\title{
Mitochondria-mediated defense mechanisms against pathogens in Caenorhabditis elegans
}

\author{
Sujeong Kwon ${ }^{1, \#}$, Eun Ji E. Kim ${ }^{1, \#} \mathcal{E}$ Seung-Jae V. Lee , $^{1, *}$ \\ ${ }^{1}$ Department of Life Sciences and ${ }^{2}$ Information Technology Convergence Engineering, Pohang University of Science and Technology, \\ Pohang 37673, Korea
}

\begin{abstract}
Mitochondria are crucial organelles that generate cellular energy and metabolites. Recent studies indicate that mitochondria also regulate immunity. In this review, we discuss key roles of mitochondria in immunity against pathogen infection and underlying mechanisms, focusing on discoveries using Caenorhabditis elegans. Various mitochondrial processes, including mitochondrial surveillance mechanisms, mitochondrial unfolded protein response $\left(\mathrm{UPR}^{\mathrm{mt}}\right)$, mitophagy, and reactive oxygen species (ROS) production, contribute to immune responses and resistance of $C$. elegans against pathogens. Biological processes of $C$. elegans are usually conserved across phyla. Thus, understanding the mechanisms of mitochondria-mediated defense responses in $C$. elegans may provide insights into similar mechanisms in complex organisms, including mammals. [BMB Reports 2018; 51(6): 274-279]
\end{abstract}

\section{INTRODUCTION}

Mitochondria are regarded as subcellular organelles with highly dynamic properties. Mitochondria participate in various essential cellular processes, including energy production, metabolite synthesis, and calcium homeostasis. Interestingly, recent studies indicate that mitochondria also function in unconventional processes, including immune and defense responses against pathogens and other stressors. For example, mitochondrial reactive oxygen species (mtROS) and damageassociated molecular patterns (DAMPs) can activate antibacterial and anti-viral innate immune responses in mammalian cells $(1,2)$. However, most studies on the role of mitochondria in immunity have been performed using

*Corresponding author. Tel: +82-54-279-2351; Fax: +82-54-2792199; E-mail: seungjaelee@postech.ac.kr

${ }^{\#}$ These authors contributed equally to this work.

https://doi.org/10.5483/BMBRep.2018.51.6.111

Received 26 April 2018

Keywords: Caenorhabditis elegans, Immunity, Mitochondria, Pathogen, Surveillance cultured cells but rarely using live organisms.

Caenorhabditis elegans is an outstanding model organism to study innate immunity for several reasons $(3,4)$. First, diverse bacteria such as Pseudomonas aeruginosa and Staphylococcus aureus that infect $C$. elegans are also human pathogens. Second, C. elegans is genetically tractable for identifying key immune factors. Third, assays that measure the death of $C$. elegans on pathogens, the ultimate outcome of infection and impaired immunity, are rapid and inexpensive. Fourth, changes in both host and pathogen can be simultaneously monitored. Fifth, and most importantly, several key immune response pathways such as p38 mitogen-activated protein kinase (MAPK) pathway are conserved between C. elegans and mammals. Using these advantages of C. elegans, many important discoveries in the research field of innate immunity, including roles of mitochondria in organismal immunity, have been made over the last two decades.

In this review, we will discuss how mitochondrial processes, including mitochondrial surveillance mechanisms, mitochondrial unfolded protein response $\left(\mathrm{UPR}^{\mathrm{mt}}\right)$, mitophagy, and generation of reactive oxygen species (ROS), participate in C. elegans immunity. We further describe mechanisms, pathways, and types of innate immune response genes regulated by these mitochondrial processes to protect animals against pathogens and stressful environments. Information gained using $C$. elegans is generally and widely applicable to other organisms. Therefore, understanding the role of mitochondria in C. elegans immunity will provide important clues regarding mitochondrial function in immunity in mammals, including humans.

\section{MITOCHONDRIAL SURVEILLANCE}

\section{Mitochondrial surveillance is important for survival upon pathogen infection}

Mitochondria are frequent targets of virulence factors and microbial toxins as they are rich in hemes and iron-sulfur proteins (5), which are scarce in the environment but crucial for the survival of pathogens $(6,7)$. Early warning signals of mitochondrial dysfunction are used as one of the first lines of defense to initiate immune responses. Mitochondrial surveillance mechanisms constantly monitor the disruption of 
mitochondrial homeostasis and repair damaged mitochondria, thus protecting the mitochondria from various environmental stresses (8). Up-regulation of several defense-related genes, including those involved in drug-detoxification, mitochondrial repair, and response to pathogen infection, is an important part of the mitochondrial surveillance pathway. This restores mitochondrial homeostasis under environmental stresses and increases host survival (9). Overall, mitochondrial surveillance mechanisms can sense mitochondrial dysfunction and activate genes involved in mitochondrial damage repair, thus improving host immunity and its chance of survival.

\section{Conserved abiotic stress mechanism contributes to mitochondrial surveillance-mediated immunity}

Ethanol and stress response element (ESRE) network is a conserved defense mechanism in response to various abiotic stresses. Activation of ESRE gene network contributes to the immunity of $C$. elegans against $P$. aeruginosa infection through mitochondrial surveillance mechanisms (Fig. 1) (10). P. aeruginosa can utilize a variety of virulence factors to colonize and infect diverse hosts, including C. elegans and humans. ESRE is a conserved 11-nt sequence motif that is enriched in regulatory regions of genes specifically up-regulated in $C$.

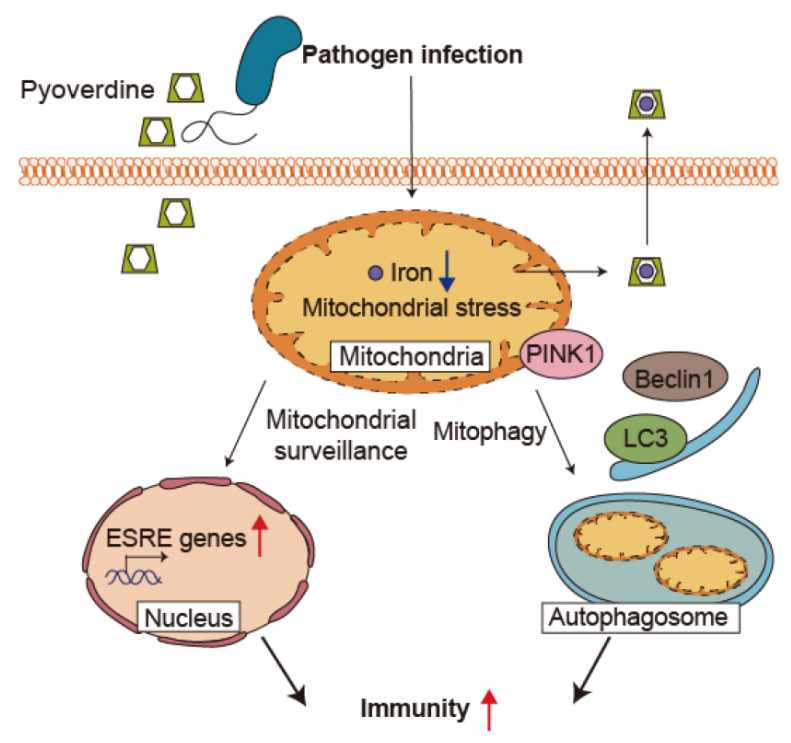

Fig. 1. Mitochondrial surveillance and mitophagy contribute to immunity. Pyoverdine, a siderophore produced by Pseudomonas aeruginosa, binds and removes iron in hosts, triggering mitochondrial stress. Host mitochondrial surveillance mechanism detects mitochondrial stress and activates ESRE (Ethanol and Stress Response Element) gene network. The induction of ESRE-containing genes increases immunity. Reduced mitochondrial iron levels also induce mitophagy that enhances immunity, acting through autophagy machinery proteins, including PTEN-induced putative kinase 1 (PINK1), Beclin 1, and microtubule-associated protein light chain 3 (LC3). elegans in response to liquid culture-based infection of $P$. aeruginosa. Importantly, the induction of ESRE-containing genes during $P$. aeruginosa infection is required for innate immune responses in C. elegans. The ESRE gene network is activated in response to pyoverdine, a virulent factor toxin and high-affinity iron chelator secreted by $P$. aeruginosa for iron uptake (11). Pyoverdine targets mitochondria and causes a hypoxia-like response in $C$. elegans, which is different from the classical innate immune response against intestinal $P$. aeruginosa colonization (10). C. elegans appear to monitor iron level and mitochondrial damage, both of which can act as signals to activate the ESRE network against $P$. aeruginosa. The induction of ESRE gene network appears to be mediated by several bZIP transcription factors whose functions are crucial for innate immune responses against $P$. aeruginosa. Overall, these studies suggest that the ESRE defense network contributes to mitochondrial surveillance which in turn promotes immunity against $P$. aeruginosa.

\section{MITOPHAGY}

Mitophagy is an autophagic process that degrades damaged mitochondria for recycling (12). One of the first processes of mitophagy is the interaction between PTEN-induced putative kinase 1 (PINK1) and cytosolic E3 ubiquitin ligase Parkin (13). The accumulation of PINK1 on damaged mitochondria is both necessary and sufficient for the recruitment of Parkin (14-17). This event also enables the autophagic machinery to distinguish between dysfunctional and healthy mitochondria. Damaged mitochondria are subsequently engulfed by autophagosomes and delivered to lysosomes for degradation (18). Mitophagy is critical for maintaining energy and calcium homeostasis and for metabolite synthesis. Recent studies have shown that the interplay between the orchestrated mitochondrial complex and mitophagy machinery is critical for immune response (19). P. aeruginosa infection disrupts mitochondrial homeostasis of $C$. elegans because of iron chelation, which triggers mitochondrial turnover (Fig. 1). This leads to mitophagy in C. elegans to cope with the loss of iron and bring the electron potential across the mitochondrial membrane back to normal. Increase in the expression of PINK1 and autophagy machinery proteins following $P$. aeruginosa infection activates mitophagy in C. elegans. This appears to be a key event for immunity because the disruption of genes required for mitophagic regulation (pink-1/PINK1 and $p d r-1 /$ Parkin) or general autophagy (bec-1/Beclin 1, lgg- $1 /$ microtubule-associated protein light chain 3 (LC3) and mboa-7/membrane-bound O-acyltransferase domain-containing protein 7$)$ significantly increases the mortality of $C$. elegans upon infection with $P$. aeruginosa (19). Thus, the induction of mitophagy is essential for enhancing resistance against pathogen infection. 


\section{MITOCHONDRIAL UNFOLDED PROTEIN RESPONSE}

\author{
Unfolded protein response (UPR) maintains protein \\ homeostasis important for cellular function and organismal \\ survival
}

UPR is an evolutionarily conserved proteostasis (protein homeostasis) system in various organisms (20-26). Multiple proteostasis pathways exist in the cytosol, endoplasmic reticulum, and mitochondria. These pathways are coordinated by and/or communicate with the nucleus. Mechanisms of heat-shock response in the cytoplasm and of UPR in the endoplasmic reticulum (UPR ${ }^{\mathrm{ER}}$ ) have been extensively studied $(21,22,24)$. UPR ${ }^{\mathrm{mt}}$ has also been recently discovered and functionally characterized $(23,26)$.

The accumulation of misfolded or unfolded proteins in the mitochondrial matrix beyond the capacity of mitochondrial chaperones triggers $U^{\mathrm{m}} \mathrm{R}^{\mathrm{mt}}$ (27). This event drives ATFS-1, a bZip transcription factor that possesses both mitochondrial and nuclear localization sequences, to translocate from mitochondria to the nucleus $(27,28)$. Subsequently, a mitochondrial matrix protease, CLPP-1, cleaves excessive proteins into short peptides (29). HAF-1, an ATP-binding cassette (ABC) transporter, then exports these small peptides from mitochondria to the cytosol and slows mitochondrial import of ATFS-1, further assisting its nuclear localization (27, 28). Nuclear localized ATFS-1 then transactivates mitochondrial protective genes, including those encoding mitochondrial chaperones that help mitochondrial proteostasis, and anti-microbial genes $(27,30,31)$.

Exposure to $P$. aeruginosa is one of the stimuli that cause mitochondrial stress and activate UPR ${ }^{\mathrm{mt}}$ in C. elegans (30). $P$. aeruginosa accumulates in the intestine of worms upon infection and causes mitochondrial stress in intestinal cells (Fig. 2). Increased expression of mitochondrial chaperone genes and ATFS-1 translocation to nuclei are detected during $P$. aeruginosa infection. Subsequently, nuclear localized ATFS-1 induces the expression of innate immunity genes in addition to mitochondrial protective genes to cope with the infection. Thus, utilizing UPR ${ }^{\mathrm{mt}}$ through ATFS- 1 is an important means to detect pathogen infection by sensing impaired mitochondria and initiating innate immune responses in $C$. elegans.

\footnotetext{
Mitochondrial chaperone HSP-60 enhances innate immunity against pathogenic bacteria

We have previously performed genetic screening of over 200 evolutionarily conserved mitochondrial components to identify novel mitochondrial factors that affect C. elegans innate immunity against $P$. aeruginosa (32). Our subsequent functional analysis has shown that genetic inhibition of HSP-60, one of candidates identified from the screen, drastically reduces the chance of survival of $P$. aeruginosa-infected worms. HSP-60 is a nuclear encoded mitochondrial chaperone and a key component of $\operatorname{UPR}^{\mathrm{mt}}(23,26)$. Mitochondrial
}

chaperones including HSP-60 can reduce the level of misfolded or unfolded proteins and help maintain mitochondrial proteostasis. We have shown that HSP-60 acts in intestinal cells and neurons to enhance the innate immunity of $C$. elegans against $P$. aeruginosa (32). Furthermore, HSP-60 has a small effect on the lifespan and pathogen avoidance behavior of $C$. elegans, suggesting that HSP-60 can specifically increase innate immunity against $P$. aeruginosa.

\section{HSP-60 up-regulates p38 MAPK/PMK-1 signaling to enhance immunity}

The main immune signaling pathways in C. elegans include PMK-1 (p38 mitogen-activated protein kinase (MAPK)), ZIP-2, SKN-1/Nrf, and DAF-16/FOXO pathways (3, 4, 33). HSP-60 increases innate immunity against $P$. aeruginosa in a PMK-1-dependent manner (Fig. 2). However, HSP-60mediated innate immunity is independent of other immune regulators such as ZIP-2, SKN-1/Nrf, and DAF-16/FOXO (32). PMK-1 is activated via phosphorylation by upstream kinases that are important for resistance against $P$. aeruginosa. HSP-60 appears to contribute to the maintenance of active

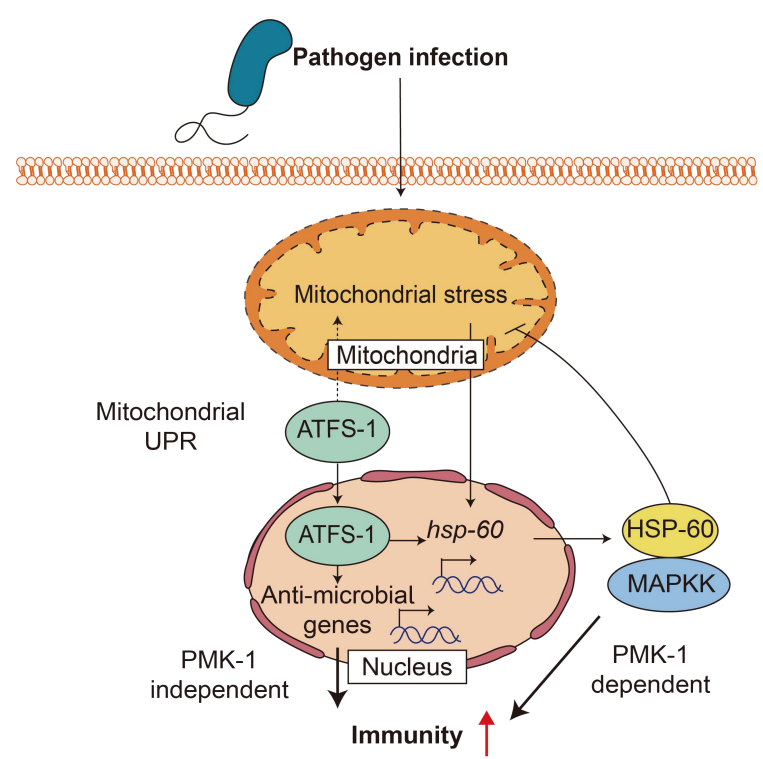

Fig. 2. Mitochondrial unfolded protein response mediates C. elegans immunity. Pathogen infection causes mitochondrial stress and activates mitochondrial unfolded protein response $\left(\mathrm{UPR}^{\mathrm{mt}}\right)$ in C. elegans. ATFS-1, a key UPR ${ }^{\mathrm{mt}}$ regulatory transcription factor, is translocated to the nucleus to induce anti-microbial genes and mitochondrial chaperones. Mitochondrial chaperone HSP-60 directly binds to cytosolic mitogen-activated protein kinase kinase (MAPKK), which up-regulates p38 mitogen-activated protein kinase (PMK-1) immune signaling. 
phosphorylated PMK-1 by increasing the level of upstream MAPKK. Interestingly, a large fraction of HSP-60 is localized in the cytosol as well as the mitochondria. The cytosolic HSP-60 physically binds to MAPKK and increases resistance against $P$. aeruginosa via stabilizing MAPKK. Overall, our findings suggest that HSP-60, previously known as a mitochondrial chaperone, can act through cytosolic PMK-1 signaling to enhance innate immunity in C. elegans.

\section{MITOCHONDRIAL ROS}

\section{ROS act as signaling molecules for physiology}

ROS, such as superoxide, hydroxyl radical, hydrogen peroxide, and singlet oxygen, are highly reactive and toxic molecules $(34,35)$. Mitochondria generate high amounts of ROS as byproducts of the electron transport chain during oxidative phosphorylation. Various cellular stimuli including cytokines, hypoxia, and increased calcium concentration regulate levels of mtROS. mtROS are not only toxic byproducts but also play key roles in various cellular processes, including cell differentiation, autophagy, metabolic adaptation, longevity, and immunity.

A feedback mechanism regulates mtROS-mediated immunity We have previously shown that mtROS can increase resistance against pathogenic bacteria via hypoxia-inducible factor 1

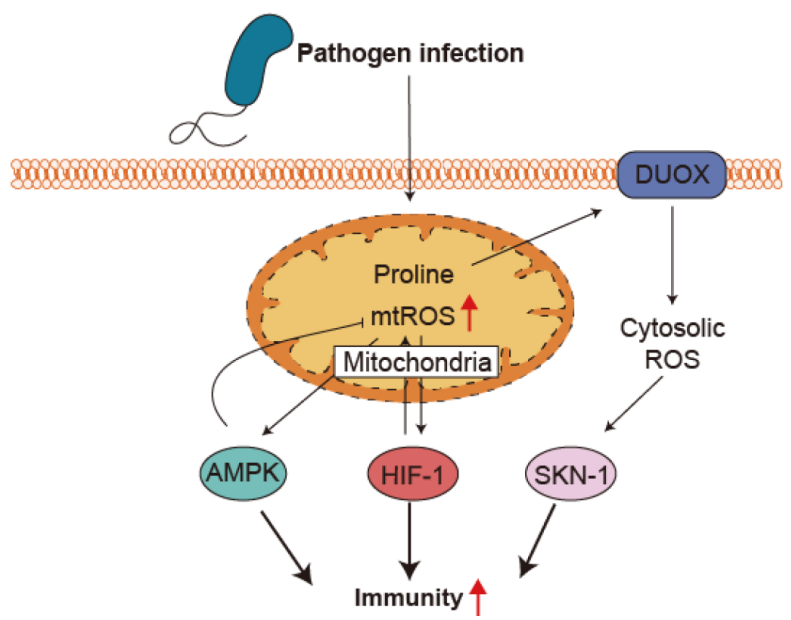

Fig. 3. Mitochondrial reactive oxygen species enhance immunity. Upon pathogen infection, mitochondrial ROS (mtROS) increase immunity in C. elegans. AMP-activated protein kinase (AMPK) and hypoxia-inducible factor 1 (HIF-1) mediate mtROS-induced immunity through negative and positive feedback mechanisms, respectively. Mitochondrial proline catabolism also regulates ROS levels and immunity. The intermediate metabolite produces cytosolic ROS by dual oxidase (DUOX) and activates SKN-1/Nrf, an important immune regulator. Proper communication between mitochondrial and cytosolic ROS leads to enhanced immunity against $P$. aeruginosa.
(HIF-1) and AMP-activated protein kinase (AMPK) (36) (Fig. 3). Mild inhibition of mitochondrial respiration has been shown to increase lifespan and the level of $\operatorname{mtROS}(37,38)$. We have shown that increased mtROS levels up-regulate AMPK and HIF-1, both of which are important mediators of longevity (36). HIF-1 has a conserved AMPK recognition motif that is directly phosphorylated by AMPK. Interestingly, AMPK and HIF-1 mutually down-regulate each other. This appears to be important for equilibrating signaling between these two factors.

AMPK and HIF-1 also regulate mtROS levels through feedback mechanisms. AMPK inhibits mtROS production in a negative feedback manner whereas HIF-1 increases mtROS production through positive feedback regulation. Together, AMPK and HIF-1 maintain mtROS at an optimal level to increase innate immunity against various pathogenic bacteria, including $P$. aeruginosa, Enterococcus faecalis, and pathogenic $E$. coli (36). In contrast to HIF-1 and AMPK, other known factors regulating the longevity of mitochondrial respiration mutants that contain high ROS levels have small or no effects on innate immunity. Therefore, the regulation of appropriate mtROS levels through feedback mechanisms involving AMPK and HIF-1 enhances innate immunity.

\section{Mitochondrial proline catabolism regulates innate immunity via $R O S$ and SKN-1/Nrf in C. elegans}

Treatment of $C$. elegans with proline increases its innate immunity against $P$. aeruginosa (39) (Fig. 3). Proline dehydrogenase $(\mathrm{PRODH})$ catabolizes proline to pyrroline-5-carboxylate (P5C). PRODH is required for proline-induced immunity by increasing ROS levels and for the activation of SKN-1/Nrf, an important transcription factor for innate immunity and detoxification. Increasing proline and PRODH levels leads to the accumulation of $\mathrm{P} 5 \mathrm{C}$, which acts as a signal for ROS production and $\mathrm{SKN}-1 / \mathrm{Nrf}$ activation in response to $P$. aeruginosa infection. Additionally, BLIS-3/DUOX, a plasma membrane-localized NADPH oxidase/dual-oxidase, is another major source of ROS production. BLIS-3/DUOX is required for proline-induced immune response. Cytosolic ROS produced by BLIS-3/DUOX subsequently attack pathogenic bacteria and increase protective SKN-1/Nrf activity in a p38 MAPK-dependent manner in the intestine (40-42). Thus, communication between mitochondria and plasma membrane via ROS contributes to resistance of $C$. elegans against pathogenic bacteria. In addition to these findings, epidermal wounding generates mtROS in response to $\mathrm{Ca}^{2+}$ influx during wound closure (43). Increased mtROS play a role in wound healing via regulating actin ring closure. Together, these studies suggest that mtROS play critical roles in defense mechanisms such as anti-bacterial immunity and wound healing in C. elegans.

\section{CONCLUSIONS}

Because mitochondria have very important cellular functions, alterations or defects in mitochondrial activity will adversely 
affect energy metabolism, development, health span, and lifespan in various organisms $(26,44-46)$. However, the role of mitochondria in immunity has only been recently established. Molecular genetic studies in C. elegans have answered many important research questions. Mitochondrial surveillance mechanisms such as UPR ${ }^{\mathrm{mt}}$, mitophagy, and ROS production are recently discovered mitochondrial processes that participate in $C$. elegans immune response against pathogen infection. These mitochondrial processes utilize different types of established immune pathways, such as p38 MAPK/PMK-1, PINK1, HIF-1, AMPK, and SKN-1/Nrf signaling pathways.

The mitochondrial processes covered in this review are evolutionarily well conserved in diverse species. Therefore, findings in C. elegans immunity also have implications in other species, including mammals. Further studies on the network of mitochondrial processes and defense mechanisms are needed to assist the development of novel therapeutic interventions that can improve pathogen resistance and health in humans.

\section{ACKNOWLEDGEMENTS}

We thank the Lee laboratory members for helpful discussion on the manuscript.

This work was supported by a grant [NRF-2016R1E1A 1A01941152 to S-J.V.L.] of the National Research Foundation (NRF) funded by the Ministry of Science, ICT and Future Planning (MSIP), Republic of Korea.

\section{CONFLICTS OF INTEREST}

The authors have no conflicting interests.

\section{REFERENCES}

1. West AP, Shadel GS and Ghosh S (2011) Mitochondria in innate immune responses. Nat Rev Immunol 11, 389-402

2. Mehta MM, Weinberg SE and Chandel NS (2017) Mitochondrial control of immunity: beyond ATP. Nat Rev Immunol 17, 608

3. Ewbank JJ and Pujol N (2016) Local and long-range activation of innate immunity by infection and damage in C. elegans. Curr Opin Immunol 38, 1-7

4. Kim DH and Ewbank JJ (2015) Signaling in the innate immune response. WormBook, 1-51

5. Prasai K (2017) Regulation of mitochondrial structure and function by protein import: A current review. Pathophysiology 24, 107-122

6. Gerwien F, Skrahina V, Kasper L, Hube B and Brunke S (2018) Metals in fungal virulence. FEMS Microbiol Rev 42

7. Chandrangsu P, Rensing C and Helmann JD (2017) Metal homeostasis and resistance in bacteria. Nat Rev Microbiol 15, 338-350

8. Shore DE and Ruvkun G (2013) A Cytoprotective Perspective on Longevity Regulation. Trends Cell Biol 23, 409-420
9. Liu Y, Samuel BS, Breen PC and Ruvkun G (2014) Caenorhabditis elegans pathways that surveil and defend mitochondria. Nature 508, 406-410

10. Tjahjono E and Kirienko NV (2017) A conserved mitochondrial surveillance pathway is required for defense against Pseudomonas aeruginosa. PLoS Genet 13, e1006876

11. Kang $\mathrm{D}$, Kirienko DR, Webster $\mathrm{P}$, Fisher $\mathrm{AL}$ and Kirienko NV (2018) Pyoverdine, a siderophore from Pseudomonas aeruginosa, translocates into C. elegans, removes iron, and activates a distinct host response. Virulence, 1-41

12. Pickles S, Vigié P and Youle RJ (2018) Mitophagy and Quality Control Mechanisms in Mitochondrial Maintenance. Curr Biol 28, R170-R185

13. Mouton-Liger $\mathrm{F}$, Jacoupy $\mathrm{M}$, Corvol J-C and Corti O (2017) PINK1/Parkin-Dependent Mitochondrial Surveillance: From Pleiotropy to Parkinson's Disease. Front Mol Neurosci 10, 120

14. Narendra DP, Jin SM, Tanaka A et al (2010) PINK1 is selectively stabilized on impaired mitochondria to activate Parkin. PLoS Biol 8, e1000298

15. Yang Y, Gehrke S, Imai Y et al (2006) Mitochondrial pathology and muscle and dopaminergic neuron degeneration caused by inactivation of Drosophila Pink1 is rescued by Parkin. Proc Natl Acad Sci U S A 103, 10793-10798

16. Clark IE, Dodson MW, Jiang C et al (2006) Drosophila pink1 is required for mitochondrial function and interacts genetically with parkin. Nature 441, 1162-1166

17. Park J, Lee SB, Lee $S$ et al (2006) Mitochondrial dysfunction in Drosophila PINK1 mutants is complemented by parkin. Nature 441, 1157-1161

18. Harper JW, Ordureau A and Heo JM (2018) Building and decoding ubiquitin chains for mitophagy. Nat Rev Mol Cell Biol 19, 93-108

19. Kirienko NV, Ausubel FM and Ruvkun G (2015) Mitophagy confers resistance to siderophore-mediated killing by Pseudomonas aeruginosa. Proc Natl Acad Sci U S A 112,1821

20. Jovaisaite V, Mouchiroud L and Auwerx J (2014) The mitochondrial unfolded protein response, a conserved stress response pathway with implications in health and disease. J Exp Biol 217, 137

21. Hetz C and Papa FR (2018) The Unfolded Protein Response and Cell Fate Control. Mol Cell 69, 169-181

22. Powers ET and Balch WE (2013) Diversity in the origins of proteostasis networks - a driver for protein function in evolution. Nat Rev Mol Cell Biol 14, 237

23. Shpilka T and Haynes CM (2018) The mitochondrial UPR: mechanisms, physiological functions and implications in ageing. Nat Rev Mol Cell Biol 19, 109-120

24. Frakes $A E$ and Dillin A (2017) The UPR ${ }^{E R}$ Sensor and Coordinator of Organismal Homeostasis. Mol Cell 66, 761-771

25. Sala AJ, Bott LC and Morimoto RI (2017) Shaping proteostasis at the cellular, tissue, and organismal level. J Cell Biol 216, 1231-1241

26. Moehle EA, Shen K and Dillin A (2018) Mitochondrial Proteostasis in the Context of Cellular and Organismal Health and Aging. J Biol Chem [Epub ahead of print] 
27. Nargund AM, Pellegrino MW, Fiorese CJ, Baker BM and Haynes CM (2012) Mitochondrial import efficiency of ATFS-1 regulates mitochondrial UPR activation. Science 337, 587-590

28. Haynes CM, Yang Y, Blais SP, Neubert TA and Ron D (2010) The matrix peptide exporter HAF-1 signals a mitochondrial UPR by activating the transcription factor ZC376.7 in C. elegans. Mol Cell 37, 529-540

29. Haynes CM, Petrova K, Benedetti C, Yang Y and Ron D (2007) ClpP mediates activation of a mitochondrial unfolded protein response in C. elegans. Dev Cell 13, 467-480

30. Pellegrino MW, Nargund AM, Kirienko NV, Gillis R, Fiorese CJ and Haynes CM (2014) Mitochondrial UPR-regulated innate immunity provides resistance to pathogen infection. Nature 516, 414-417

31. Nargund AM, Fiorese CJ, Pellegrino MW, Deng P and Haynes CM (2015) Mitochondrial and nuclear accumulation of the transcription factor ATFS-1 promotes OXPHOS recovery during the UPR(mt). Mol Cell 58, 123-133

32. Jeong DE, Lee D, Hwang SY et al (2017) Mitochondrial chaperone HSP-60 regulates anti-bacterial immunity via p38 MAP kinase signaling. EMBO J 36, 1046-1065

33. Cohen LB and Troemel ER (2015) Microbial pathogenesis and host defense in the nematode $C$. elegans. Curr Opin Microbiol 23, 94-101

34. Van Raamsdonk JM and Hekimi S (2010) Reactive Oxygen Species and Aging in Caenorhabditis elegans: Causal or Casual Relationship? Antioxid Redox Signal 13, 1911-1953

35. Hwang AB and Lee SJ (2011) Regulation of life span by mitochondrial respiration: the HIF-1 and ROS connection. Aging (Albany NY) 3, 304-310

36. Hwang $A B$, Ryu EA, Artan $M$ et al (2014) Feedback regulation via AMPK and HIF-1 mediates ROS-dependent longevity in Caenorhabditis elegans. Proc Natl Acad Sci U
S A 111, E4458-4467

37. Lee SJ, Hwang AB and Kenyon C (2010) Inhibition of respiration extends $C$. elegans life span via reactive oxygen species that increase HIF-1 activity. Curr Biol 20, 2131-2136

38. Yang W and Hekimi S (2010) A Mitochondrial Superoxide Signal Triggers Increased Longevity in Caenorhabditis elegans. PLoS Biol 8, e1000556

39. Tang $\mathrm{H}$ and Pang S (2016) Proline Catabolism Modulates Innate Immunity in Caenorhabditis elegans. Cell Rep 17, 2837-2844

40. Chavez V, Mohri-Shiomi A and Garsin DA (2009) Ce-Duox1/BLI-3 generates reactive oxygen species as a protective innate immune mechanism in Caenorhabditis elegans. Infect Immun 77, 4983-4989

41. van der Hoeven R, McCallum KC, Cruz MR and Garsin DA (2011) Ce-Duox1/BLI-3 Generated Reactive Oxygen Species Trigger Protective SKN-1 Activity via p38 MAPK Signaling during Infection in C. elegans. PLoS Pathog 7, e1002453

42. McCallum KC and Garsin DA (2016) The Role of Reactive Oxygen Species in Modulating the Caenorhabditis elegans Immune Response. PLoS Pathog 12, e1005923

43. Xu S and Chisholm AD (2014) C. elegans epidermal wounding induces a mitochondrial ROS burst that promotes wound repair. Dev Cell 31, 48-60

44. Koliaki C and Roden M (2016) Alterations of Mitochondrial Function and Insulin Sensitivity in Human Obesity and Diabetes Mellitus. Annu Rev Nutr 36, 337-367

45. Kauppila TES, Kauppila JHK and Larsson NG (2017) Mammalian Mitochondria and Aging: An Update. Cell Metab 25, 57-71

46. Lima A, Burgstaller J, Sanchez-Nieto JM and Rodriguez TA (2018) The Mitochondria and the Regulation of Cell Fitness During Early Mammalian Development. Curr Top Dev Biol 128, 339-363 